\title{
The age of the main population of the Sagittarius dwarf spheroidal galaxy
}

\section{Solving the "M giant conundrum"}

\author{
M. Bellazzini ${ }^{1}$, M. Correnti ${ }^{2}$, F. R. Ferraro ${ }^{2}$, L. Monaco $^{3}$, and P. Montegriffo ${ }^{1, \star}$ \\ 1 INAF - Osservatorio Astronomico di Bologna, Via Ranzani 1, 20127 Bologna, Italy \\ e-mail: [michele.bellazzini; paolo.montegriffo]@abo.astro.it \\ 2 Dipartimento di Astronomia, Università di Bologna, Via Ranzani 1, 20127 Bologna, Italy \\ e-mail: [francesco.ferraro3; matteo.correnti]@studio.unibo.it \\ 3 ESO - European Southern Observatory, Alonso de Cordova 3107, Santiago 19, Chile \\ e-mail: lmonaco@eso.org
}

Received 15 September 2005 / Accepted 3 December 2005

\section{ABSTRACT}

We present a statistically decontaminated Color Magnitude Diagram of a $1^{\circ} \times 1^{\circ}$ field in the core of the Sagittarius dSph galaxy. Coupling this CMD with the most recent metallicity distributions obtained from high resolution spectroscopy we derive robust constraints on the mean age of the stellar population that dominates the galaxy (Pop A). Using three different sets of theoretical isochrones in the metallicity range $-0.4 \leq[\mathrm{M} / \mathrm{H}] \leq-0.7$ and taking into consideration distance moduli in the range $16.90 \leq(m-M)_{0} \leq 17.20$ we find that the mean age of Pop A is larger than $5 \mathrm{Gyr}$, and the best-fit value is age $=8.0 \pm 1.5 \mathrm{Gyr}$. Since Pop A provides the vast majority of the M giants that traces the tidal stream of Sgr dSph all over the sky, our estimate resolves the so called "M giant conundrum" (Majewski et al. 2003, ApJ, 599, 1082). The time needed by the $\mathrm{M}$ giants that currently populates the stream to diffuse within the main body of Sgr and to reach the extremes of the tidal tails once torn apart from the parent galaxy $(\simeq 3-4 \mathrm{Gyr})$ can be easily accommodated into the time lapsed since their birth $(\simeq 5.5-9.5 \mathrm{Gyr})$.

Key words. Galaxies: dwarf - Galaxies: evolution - stars: abundances

\section{Introduction}

The Sagittarius dwarf spheroidal galaxy (Sgr dSph; Ibata et al. 1994) currently provides the cleanest possibility to study in detail the tidal disruption and the accretion of a dwarf satellite into a large galaxy. The tidal tails of the disrupting galaxy have been observed in widely different positions with different tracers (see, for instance, Newberg et al. 2002; Ibata et al. 2001, and references therein). In particular, Majewski et al. (2003, hereafter M03) showed that M giants traces the tidal tails of Sgr as a coherent and dynamically cold filamentary structure (hereafter Sgr Stream) extending for tens of $\mathrm{kpc}$ from the parent galaxy and nicely aligned along the rosetta orbit of Sgr (see also Law et al. 2005).

The study of the physical properties (age and chemical abundances) and the kinematics of stars into the main body of Sgr and into the Stream may provide for the first time the possibility to link the Star Formation History (SFH) of the galaxy with its "dynamical/orbital" history and evolution (see Bellazzini et al. 1999b, hereafter BFBb) and/or to constrain

^ Based on observations collected at the European Southern Observatory, Chile, (Programme 71.D-0222A). the one with the other. A first relevant case of this interlacing was pointed out by M03. These authors obtained rough estimates of the metallicity of $M$ giants in the Sgr Stream from their $J-K$ colors and using the Age-Metallicity Relation (AMR) for Sgr provided by Layden \& Sarajedini (2000, hereafter LS00), they concluded that Stream M giants should be younger than $5 \mathrm{Gyr}$ and a significant fraction of them have an age of 2-3 Gyr. The $N$-body models that best reproduces the Sgr galaxy + Stream system (Law et al. 2005) show that a comparable amount of time is needed to produce tidal tails of the observed extension. According to these models, we are observing in the Stream $M$ giants that were torn apart from their parent galaxy up to $3.2 \mathrm{Gyr}$ ago. Since it is reasonable to imagine that in a spheroidal system star formation episodes occur preferentially toward the center of the galaxy, the above time lapse should be (possibly) increased by the time needed to the newly born stars to diffuse out to the "edges" of the system (M03). Hence, the comparison between the stellar evolution timescales (e.g., the age of M giants) and the dynamical timescales (e.g., the time needed to populate the whole extension of the Stream) give rise to a possible inconsistency, or at least a fine-tuning problem, since stars cannot be torn apart from a galaxy before 
their birth. M03 dubbed this apparent mismatch between the two timescales as "the M giants conundrum".

However, the method adopted by M03 to estimate the age of Sgr M giants is prone to uncertainties both in the previously established Sgr AMR as well as in the predicted colors of RGB tip stars from theoretical isochrones. An age estimate based on more reliable age-sensitive observables (such as the Main Sequence Turn Off - TO - and/or the Sub Giant Branch, SGB) would greatly help in clarifying the issue. This kind of estimate can be performed only in the main body of the galaxy, where the stellar density is sufficient to allow the derivation of well populated Color Magnitude Diagrams (CMD) from the observation of reasonably small fields $\left(\gtrsim 0.1 \mathrm{deg}^{2}\right)$. In this Letter we use a new large photometric dataset of Sgr stars to obtain a robust estimate of the mean age of the population that dominates the stellar content of Sgr, shedding a new light on the $\mathrm{M}$ giant conundrum.

\subsection{The dataset}

We have obtained $B, V, I$ photometry of a $1^{\circ} \times 1^{\circ}$ field located at $(l, b) \simeq\left(6.5^{\circ},-16.5^{\circ}\right), \sim 2^{\circ}$ to the East of the galaxy center, along the major axis (Sgr34 Field, see Bellazzini et al. 1999a, hereafter BFBa). The field was imaged with a mosaic of four pointings of the WFI camera mounted at the ESO/MPI $2.2 \mathrm{~m}$ telescope at La Silla, Chile. The data reduction was performed as in Monaco et al. (2002, hereafter Mo02) and the absolute photometric calibration was achieved with repeated observations of Landolt's (1993) standard fields. All the details of the data acquisition and reduction will be described in a future contribution (Bellazzini et al., in preparation). A $0.5^{\circ} \times 0.5^{\circ}$ field sampling the Galactic population at similar angular distance from the Galactic Center (Gal Field, at $(l, b) \simeq\left(-6.0^{\circ},-14.5^{\circ}\right)$ ) was also observed with the same camera, to perform the statistical decontamination of the Sgr34 CMD from the foreground/background Galactic Stars (see BFBb). The interstellar reddening was interpolated for each star from the Schlegel et al. (1998) maps and corrected according to Bonifacio et al. (2000). We found that the reddening variation over the considered fields are negligible (with standard deviations $<0.01 \mathrm{mag}$ ) and we adopted the average reddening values $E(B-V)=0.116$ for Sgr34 and $E(B-V)=0.096$ for Gal Field.

The statistically decontaminated CMD obtained from the above described datasets is presented in Fig. 1. The statistical decontamination has been performed as in $\mathrm{BFBb}$. The large samples available for both Sgr34 (more than 300000 stars) and for Gal Field ( $\simeq 57000$ stars) ensure a reliable and clean recovery of all the main features of the CMD, most of which have been labeled in Fig. 1 according to the nomenclature introduced in BFBa and Mo02. In the present context, the most relevant features of Fig. 1 are the well identified TO point at $V_{\mathrm{TO}}=21.4 \pm 0.15$ and the single, narrow and well defined SGB that is clearly visible at $V \simeq 20.8$ and $B-V \simeq 0.85$. In the following we will use these observables to constrain the mean age of Sgr.

\section{The mean age of Sgr dSph}

The availability of a large and complete sample of Horizontal Branch (HB) stars allows an accurate census of the stars older

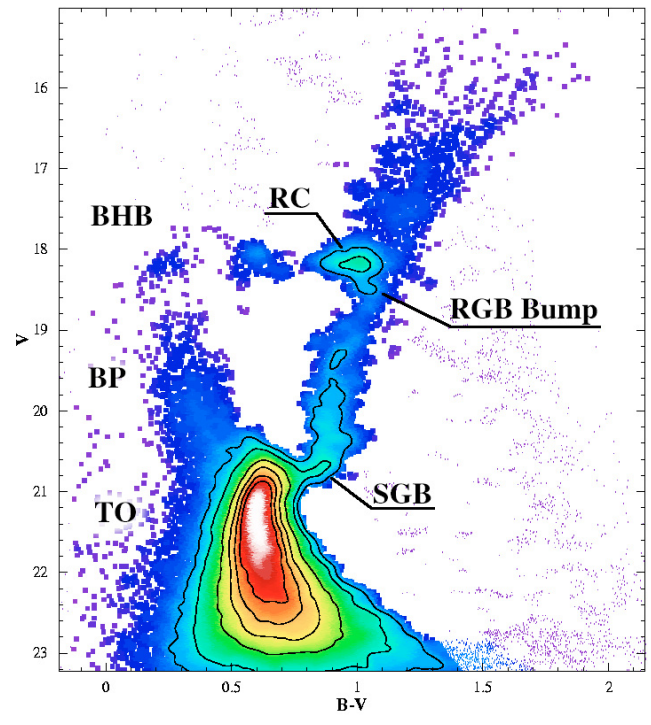

Fig. 1. Statistically decontaminated CMD of the Sgr34 Field. The color of the stars is coded according to the local density of stars on the diagram. A few representative density contours are also overplotted. Some remarkable features of the CMD are labeled: the Red Clump (RC), the Blue Horizontal Branch (BHB), the RGB Bump, the Blue Plume (BP), the Main Sequence Turn Off point (TO) and the Sub Giant Branch (SGB). The spurious residuals of the statistic decontamination process (mainly due to edge effects) to the lower left and the upper right of the main locus of the Sgr populations in the CMD have been plotted as small points to provide a clearer view of the most significant parts of the diagram.

than $\sim 1-2$ Gyr in a given stellar system. With this method Monaco et al. (2003) established that $\sim 12 \%$ of Sgr stars in this age range belong to a population with age $\gtrsim 10 \mathrm{Gyr}$ and metallicity $[\mathrm{Fe} / \mathrm{H}] \lessgtr-1.3$ (traced by BHB and RR Ly stars, a result confirmed also with the present sample). Apart from these old and metal poor stars and a very sparse (possibly young) population traced by the BP accounting for $\$ 6 \%$ of the whole stellar content (BFBa), more than $80 \%$ of Sgr stars belong to a metalrich and intermediate-old age population (Pop A, after BFBb and Mo02; see also LS00 and references therein). Observations of the RGB in the Sgr core (Cole 2001, M03) show that the dominant RGB sequence - which must correspond to Pop A includes a prominent population of $\mathrm{M}$ giants at its tip. It is logical to conclude that the M giants observed in the Sgr tails come from this population (rather than from, e.g., the much smaller and presumably even younger BP population). Thus, constraining the age of Pop A would then mean to constrain the age of $\mathrm{M}$ giants in the main body, and therefore the Stream, of Sgr. The compact morphology of the Red Clump and of the TO region and the presence of a single and well defined RGB Bump (Mo02) strongly suggest that Pop A stars spans a relatively limited age and metallicity range. The most likely SFH proposed by $\mathrm{BFBb}$ indicates that the episode that lead to the formation of Pop A lasted $\sim 3$ Gyr. Joining the samples of abundances of bona fide Sgr stars obtained with high resolution spectroscopy by Bonifacio et al. (2004, B04) and by Monaco et al. (2005, M05) we obtain a metallicity distribution from 27 stars that 


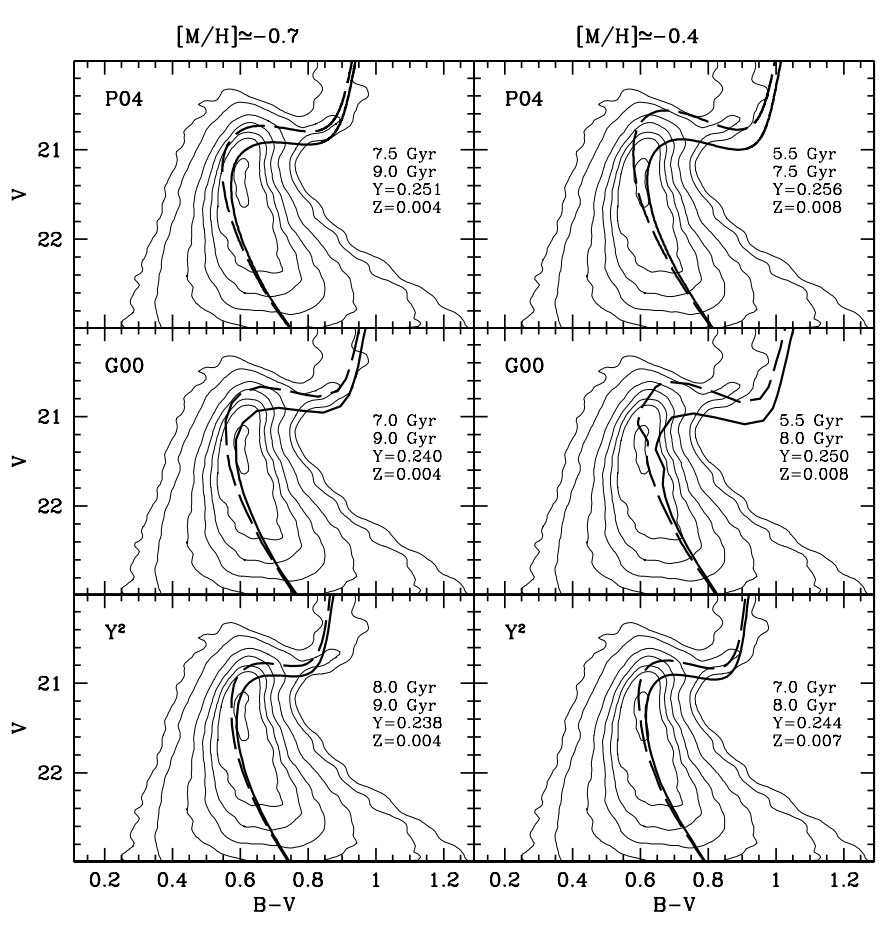

Fig. 2. Isochrone fitting of the TO/SGB region of the Sgr34 CMD, here represented with the isodensity contours shown in Fig. 1. The isochrones plotted as continuous lines provides the best fit to the TO luminosity, those plotted as dashed lines provides the best fit to the luminosity of the SGB. The left panels show the comparison with $Z=0.004$ isochrones, the right panel show the comparison with $Z=0.007-0.008$ isochrones. Isochrones from the P04 set (upper panels), G00 set (middle panels), and $\mathrm{Y}^{2}$ set (lower panels) are considered. The age, metallicity and helium content $(Y)$ of the adopted isochrones are reported in each panel.

shows a strong peak around $[\mathrm{M} / \mathrm{H}] \simeq-0.5^{1}$. The average and standard deviation of the distribution for $[\mathrm{M} / \mathrm{H}] \geq-1.2(26$ of the 27 stars of the sample) are respectively $[\mathrm{M} / \mathrm{H}]=-0.55$ and $\sigma=0.22$ dex. A preliminary analysis of the much larger (114 stars) sample described by Zaggia et al. (2004) fully confirms the essence of this result: the distribution shows a clear peak at $[\mathrm{M} / \mathrm{H}] \sim-0.6$ with the same $\sim 0.25$ dex dispersion. All the above considerations imply that a quantity as the "mean age" of Pop A is well defined and can be estimated from the CMD of Fig. 1.

In Fig. 2 we compare the TO-SGB region of the decontaminated CMD with theoretical isochrones. To account for the metallicity range spanned by the bulk of Pop A stars we consider isochrones with $Z=0.004$ and $Z \simeq 0.008$. To limit the

\footnotetext{
${ }^{1}$ All over this letter we will use the global metallicity parameter as defined by Salaris, Chieffi \& Straniero (1993), $[\mathrm{M} / \mathrm{H}]=$ $[\mathrm{Fe} / \mathrm{H}]+\log \left(0.638 \times 10^{[\alpha / \mathrm{Fe}]}+0.362\right)$. The average of the $[\mathrm{Mg} / \mathrm{Fe}]$ and $[\mathrm{Ca} / \mathrm{Fe}]$ abundances are used as a proxy of $[\alpha / \mathrm{Fe}]$ (see Monaco et al. 2005).To obtain a final sample as homogeneous as possible, we have recomputed the abundances of B04 stars from the published equivalent widths, adopting the same temperature scale (i.e. photometry and reddening) and atomic parameters used by M05. This lead to only minor revisions of the B04 estimates (i.e. an average shift of $-0.17 \pm 0.04 \mathrm{dex}$ ), removing the small offset between the two scales noted in M05.
}

sensitivity on the various assumptions influencing the stellar evolution models we perform the comparison with three different sets of isochrones, namely those by Pietrinferni et al. (2004, P04), by Girardi et al. (2000, G00), and the Yale-Yonsei set (Yi et al. 2001, $\left.Y^{2}\right)$. The distance modulus $\mu_{0}=(m-M)_{0}=$ 17.10 , recently obtained by Monaco et al. (2004) is adopted.

The luminosity of the TO point is the most reliable age indicator from a theoretical point of view. However, Pop A stars of different ages and metallicities, having different TO luminosity and colors, contribute to the definition of the TO point observed in Fig. 1. On the other hand the observed CMD shows that the vast majority Pop A stars have essentially the same luminosity during the SGB phase (see also Fig. 8 of BFBb, and the discussion reported there). Hence, in spite of the larger sensitivity of the SGB morphology to chemical composition and to subtleties of the stellar models, the single and narrow SGB may also provide a very useful constraint on the mean age of Pop A, since isochrones of any age and metallicity must reproduce this feature to provide an acceptable fit of the observed CMD. In each panel of Fig. 2 we plot both the isochrone that optimize the fit of the TO luminosity (continuous lines) and the isochrone that optimize the fit of the SGB luminosity (dashed lines). While the fit of the SGB provides systematically younger age estimates, the difference between the results of the two fits is always $\leq 2.0 \mathrm{Gyr}$, and typically $\simeq 1.0 \mathrm{Gyr}$. Hence the two methods gives essentially the same answers, within the uncertainties of each single estimate.

The main conclusion that can be drawn from Fig. 2 is that the mean age of Pop $A$ is $\sim 8.0 \pm 1.5$ Gyr for $Z=0.004$ and $\sim 6.5 \pm 1.5$ Gyr for $Z=0.008$, independently of the considered set of isochrones. We note that the $Z=0.004$ isochrones provide an overall better fit to the observed CMD, supporting the "older" age estimate. To provide a link to a more familiar age scale, we derived the age of the globular cluster 47 Tuc (which has $[\mathrm{M} / \mathrm{H}]=-0.66$, Ferraro et al. 1999) comparing the $Z=0.004$ isochrones with the CMD by Bellazzini et al. (2004). All the considered isochrone sets provide an age of $12-13 \mathrm{Gyr}$ for 47 Tuc, confirming that the Pop A is $\sim 5-7$ Gyr younger, in average, than the cluster (BFBb, Mo02).

To consider the impact of different assumptions about the distance modulus on our age estimate we proceeded as follows: we computed the average $V$ magnitude of the stars in the most horizontal region of the SGB, i.e. $0.66 \leq(B-V)_{0} \leq 0.70$, finding $V_{\mathrm{SGB}}^{0.68}=20.79 \pm 0.05$; we derived the same parameter from the $Z=0.004$ and $Z=0.008$ isochrones of the P04 set, so obtaining a calibration of $V_{\mathrm{SGB}}^{0.68}$ as a function of age. The theoretical $V_{\mathrm{SGB}}^{0.68}$ vs. Age relations were corrected for the reddening of Sgr34 and for different assumptions on the distance modulus, spanning from the shortest $\left(\mu_{0}=16.90\right.$, Alard 1996) to the longest $\left(\mu_{0}=17.19\right.$, LSO0) scales found in the literature (see Monaco et al. 2004 for a discussion). In Fig. 3 we compare the observed value of $V_{\mathrm{SGB}}^{0.68}$ with the theoretical predictions for different distance moduli. It is evident that even with the most extreme assumptions $\left(\mu_{0}=17.20\right.$ and $\left.Z=0.008\right)$ the lowest mean age allowed by the data is $>5.0 \mathrm{Gyr}$ while the range of the best-fit estimates is $5.5 \mathrm{Gyr} \leq$ age $\leq 9.5 \mathrm{Gyr}$. Note that a similar experiment performed using the magnitude of the TO as age indicator would lead to slightly older average ages. 

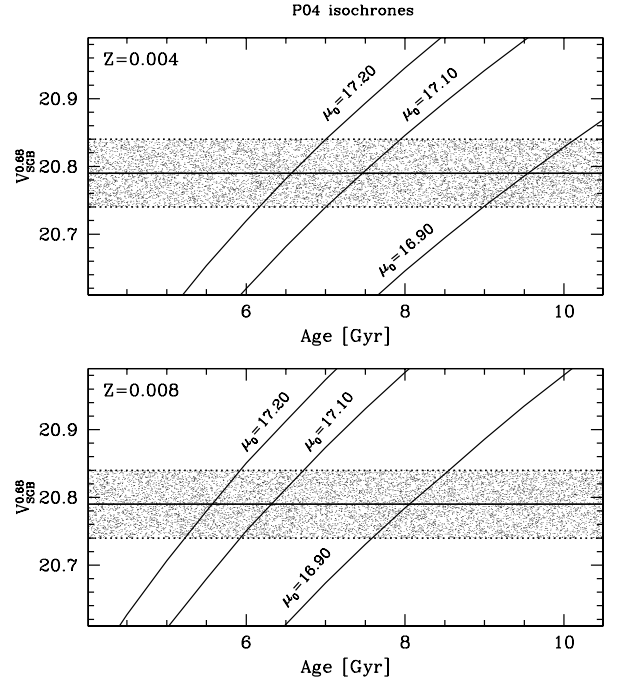

Fig. 3. Effect of varying the assumptions on the distance modulus of Sgr on the mean age estimates derived from the magnitude of the SGB at $(B-V)_{0}=0.68 \pm 0.02\left(V_{\mathrm{SGB}}^{0.68}\right)$. The continuous horizontal line marks the observed $V_{\mathrm{SGB}}^{0.68}$ level, the dotted lines enclose the range of $V_{\mathrm{SGB}}^{0.68}$ allowed by the observations, given the uncertainties (hatched). The curves labeled with different distance moduli $\left(\mu_{0}\right)$ are the theoretical predictions for $V_{\mathrm{SGB}}^{0.68}$ as a function of age computed from the P04 isochrones. Both the $Z=0.004$ (upper panel) and $Z=0.008$ (lower panel) cases are considered.

The different assumptions on distance modulus are at the origin of the younger "best-fit" ages found by LS00 with respect to the present analysis. Moreover, it should be considered that the AMR adopted by M03 for their estimate of the age of M giants in the Sgr Stream was obtained (by LS00) when essentially no spectroscopic estimate of the metallicity of Sgr stars was available. Hence, while it may provide useful general constraints on the chemical evolution history of Sgr (see LS00), the inherent uncertainties are probably too large to use it to convert metallicities into ages. For example, if we convert spectroscopic $[\mathrm{Fe} / \mathrm{H}]$ (from the M05+B04 sample discussed above) into ages using the LS00 AMR we find that $70 \%$ of the stars have an age $\leq 3.0 \mathrm{Gyr}$. This is in sharp contrast with the CMD of Sgr shown in Figs. 1 and 2, above: isochrones of ages $\leq 3.0 \mathrm{Gyr}$ would provide an exceedingly bad fit to the observed TO and SGB. Finally, the conversion of metallicities into ages with a non-linear AMR may propagate acceptable uncertainties in metallicity into large - and metallicity dependent - errors in age. With the AMR of LSO0, an error of \pm 0.2 dex in metallicity lead to a maximum error in the derived age of $\sim \pm 3.0 \mathrm{Gyr}$, at $[\mathrm{Fe} / \mathrm{H}] \sim-0.6$.

It is important to stress, here, that we are not claiming that all Pop A stars are older than 5 Gyr. It is very likely that younger stars are also there and that Pop A stars follow an AMR (Montegriffo et al. 1998, LS00). The key result of this Letter is that the majority of Pop A stars have an age sufficiently large to allow them (and the associated $\mathrm{M}$ giants) to populate the observed Stream, in agreement with the current best-fit models of the disruption of the Sgr galaxy.

\section{Conclusions}

The above described results robustly establishes that an age of $5 \mathrm{Gyr}$ is not an upper limit for the bulk of Pop A (and, consequently, of $\mathrm{M}$ giants in the main body of Sgr and in the Stream) as assumed by M03, but, in fact, a strong lower limit to the age of most of these stars. Moreover, independently of the adopted theoretical models and of the assumed distance, the best fit age for this population lies in the range $5.5 \leq$ age $\leq 9.5 \mathrm{Gyr}$, and our preferred solution $(Z=0.004$ and $\mu_{0}=17.10$, averaging over the results from the different isochrones sets) is $\langle\mathrm{age}\rangle_{\mathrm{PopA}}=8.0 \pm 1.5 \mathrm{Gyr}$. This evolutionary timescale is now comfortably larger than the dynamical timescale provided by realistic simulations of the formation of the Sgr Stream (Law et al. 2005), hence the $M$ giant conundrum appears to be solved.

Acknowledgements. This research is partially supported by the INAFPRIN2005 grant assigned to the project "A hierarchical merging tale told by stars: motions, ages and chemical compositions within structures and substructures of the Milky Way". The financial support of MIUR is also acknowledged. We are grateful to the Referee (S.R. Majewski) for insightful discussions and for very useful suggestions that improved the overall quality of the paper.

\section{References}

Alard, C. 1996, ApJ, 458, L17

Bellazzini, M., Ferraro, F. R., \& Buonanno, R. 1999a, MNRAS, 304, 633

Bellazzini, M., Ferraro, F. R., \& Buonanno, R. 1999b, MNRAS, 307, 619 (BFBb)

Bellazzini, M., Ferraro, F. R., Sollima, A., Pancino, E., \& Origlia, L. 2004, A\&A, 424, 199

Bonifacio, P., Monai, S., \& Beers, T. C. 2000, AJ, 120, 2065

Bonifacio, P., Sbordone, L., Marconi, G., Pasquini, L., \& Hill, V. 2004, A\&A, 414, 503 (B04)

Cole, A. A. 2001, ApJ, 559, L17

Ferraro, F. R., Messineo, M., Fusi Pecci, F., et al. 1999, AJ, 118, 1738

Girardi, L., Bressan, A., Bertelli, G., \& Chiosi, C. 2000, A\&AS, 141, 371

Ibata, R. A., Irwin, M. J., \& Gilmore, G. 1994, Nature, 370, 194

Ibata, R. A., Lewis, G. F., Irwin, M., Totten, E., \& Quinn, T. 2001, ApJ, 551, 294

Layden, A. C., \& Sarajedini, A. 2000, AJ, 119, 1760 (LS00)

Law, D. R., Johnston, K. V., \& Majewski, S. R. 2005, ApJ, 619, 807

Landolt, A. U. 1993, AJ, 104, 340

Majewski, S. R., Skrutskie, M. F., Weinberg, M. D., \& Ostheimer, J. C. 2003, ApJ, 599, 1082 (M03)

Monaco, L., Ferraro, F. R., Bellazzini, M., \& Pancino, E. 2002, ApJ, 578, L47

Monaco, L., Bellazzini, M., Ferraro, F. R., \& Pancino, E. 2003, ApJ, 597, L25

Monaco, L., Bellazzini, M., Ferraro, F. R., \& Pancino, E. 2004, MNRAS, 353, 874

Monaco, L., Bellazzini, M., Bonifacio, P., et al. 2005, A\&A, 441, 141 (M05)

Montegriffo, P., Bellazzini, M., Ferraro, F. R., et al. 1008, MNRAS, 294, 315

Newberg, H. J., Yanny, B., Rockosi, C., et al. 2002, ApJ, 569, 245

Pietrinferni, A., Cassisi, S., Salaris, M., \& Castelli, F. 2004, ApJ, 612, 168

Salaris, M., Chieffi, A., \& Straniero, O. 1993, ApJ, 414, 580

Schlegel, D. J., Finkbeiner, D. P., \& Davis, M. 1998, ApJ, 500, 525

Yi, S., Demarque, P., Kim, Y.-C., et al. 2001, ApJS, 136, 417

Zaggia, S., Bonifacio, P., Bellazzini, M., et al. 2004, Mem. SAIt Suppl., 5, 291 